\title{
Evaluation of joint medical and nursing notes with preprinted prompts
}

\author{
A Robins, A Gallagher, M A Rossiter, B W Lloyd
}

\begin{abstract}
Objective-To determine the views of doctors and nurses about two recent innovations in the structure of case notes: the use of preprinted prompts and the use of joint medical and nursing notes.

Design-Questionnaire survey of all doctors and nurses working on the children's wards.

Setting-Children's wards in a district general hospital.

Main outcome measures-Whether or not respondents wanted to return to traditional notes; positive and negative aspects of the two innovations.

Results-There was an $81 \%$ response rate. 45 of 48 respondents (94\%) did not want to return to traditional notes. Positive features of joint notes that were identified included: promotes team work (21/48 respondents), improves access to information (14/48), and reduces duplication (14/48). Negative features included uncertainty about identity of writer (8/48) and incompletely filled in sheets (7/48). Positive features of preprinted prompts included: less information omitted (29/48), easier to read and find information (28I $48)$, and quicker to write (21/48). Negative features included: not enough space (19/ 48) and clerking too mechanical (16/48). Conclusion-Advantages of both innovations outweighed their disadvantages to the extent that only three out of 48 respondents wanted to return to writing traditional notes.

(Quality in Health Care 1997;6:192-193)
\end{abstract}

Keywords: notes; medical and nursing; preprinted prompts

Children's

Department, North Middlesex Hospital, London, UK A Robins, paediatric senior registrar A Gallagher, clinical nurse specialist

M A Rossiter, consultant paediatrician

B W Lloyd, consultant paediatrician

Correspondence to: Dr B W Lloyd at the Children's Department, Royal Free Hospital, London NW3 2QG, UK.

Accepted for publication 30 October 1997

\section{Introduction}

Since 1993 our medical notes on admission have included preprinted prompts. We have shown that such notes are both more complete and quicker to write than are traditional notes. ${ }^{1}$

In 1994 we introduced joint medical and nursing notes. The admission notes feature preprinted prompts and cover six sides of A4 paper. A doctor and a nurse contribute to their completion. During the admission all staff write notes on standard continuation sheets. Although joint medical and nursing notes have been advocated there has been no thorough evaluation of their use.

We decided to study what the doctors and nurses who use joint notes with preprinted prompts on our wards think of them and whether or not they would prefer to return to traditional notes.

\section{Methods}

We distributed questionnaires (appendix) to the 59 doctors and nurses working on the children's wards between July and September 1995. Non-respondents were sent another questionnaire. There were 23 doctors (five consultants) and 36 nurses (six sisters).

For both innovations (joint notes and preprinted prompts) the respondent was asked to list up to three positive and up to three negative features. The respondent was then asked whether he or she would like to return to traditional notes.

\section{Results}

Forty eight of the 59 questionnaires were returned $(81 \%)$.

RESPONDENTS' VIEWS OF JOINT NOTES

Ten different positive features of joint notes were cited a total of 89 times (table 1). Nine negative features were cited a total of 30 times. The most frequently cited positive features were each cited at least twice as often as the most frequently cited negative findings.

RESPONDENTS' VIEWS OF PREPRINTED PROMPTS Twelve positive features of preprinted prompts were cited a total of 119 times (table 2). Ten negative features were cited a total of 63 times.

Table 1 Reported positive and negative features of joint notes

\begin{tabular}{ll}
\hline & $\begin{array}{l}\text { Respondents } \\
(n)\end{array}$ \\
& \\
\hline Positive features: & 21 \\
Improves doctor and nursing teamwork & 14 \\
Better access to information & 14 \\
Less duplication & 13 \\
Easier to follow progress in notes & 12 \\
Better communication and handovers & 7 \\
Less paper work and wasted paper & 5 \\
Aids decision making & 1 \\
Improved data collection & 1 \\
Good for students and trainees & 1 \\
More thoughtful notes & 8 \\
Negative features: & 7 \\
Uncertainty about the role of the writer & 4 \\
Incompletely filled in sheets & \\
Poor nursing history (inadequate care plans) & \\
Uncertainty whose job it is to fill in blank & 4 \\
spaces & 2 \\
Nurses write too much & 2 \\
Problems if doctors and nurses need them & \\
simultaneously & \\
Inadequate space to write in & 1 \\
Thoughtless comments can easily be made & 1 \\
Unfamiliar and confusing for trainees & 1 \\
\hline
\end{tabular}


Table 2 Reported positive and negative features of preprinted prompts

\begin{tabular}{ll}
\hline & $\begin{array}{l}\text { Respondents } \\
(n)\end{array}$ \\
& \\
\hline Positive features: & 29 \\
Nothing omitted & 28 \\
Easier to read and find information & 21 \\
Quicker to fill in and less writing & 13 \\
Clearer layout & 8 \\
Prompts act as reminders & 5 \\
Provides minimum data set on each child & 5 \\
Saves duplication & 3 \\
Clearer management plans & 3 \\
Easier for trainees to admit patients & 2 \\
Easy to clerk when tired & 1 \\
Looks professional & 1 \\
Good for audit & \\
Negative features: & 19 \\
Prevents thinking and clerking too & 16 \\
mechanical & \\
Not enough space for writing & 9 \\
Specific complaints about the preprinted & 6 \\
layout & 4 \\
Incompletely filled in if unprompted & 2 \\
No allergy prompt & 2 \\
Waste of paper if short admission & 2 \\
Irrelevant information collected & 2 \\
Senior doctors are quicker without prompts & 1 \\
Leads to dependence on preprinted prompts & 2 \\
Loss of details of clinical impressions & \\
\hline
\end{tabular}

Again the most frequently cited positive features were each cited considerably more often that the most frequently cited negative findings.

Forty five of the 48 respondents (94\%) wanted to retain the joint notes with preprinted prompts.

\section{Discussion}

Our study shows that our colleagues overwhelmingly endorsed the twin innovations of joint notes with preprinted prompts. This remains true even if (as seems unlikely) all 11 non-respondents wished to return to traditional notes.

It is a shortcoming of the study that there was no weighting of the degree of importance of any positive or negative feature cited by the respondents. One clue to the views of the doctors and nurses is that each positive feature was cited about twice as often as each negative feature.

Our survey shows that staff perceive joint notes as promoting team work and as being more effective than traditional notes. The potential value of joint notes has been recognised ${ }^{2}$ but we can find only one previous evaluation of their use (in a ward for elderly patients). ${ }^{3}$ This report contains few details of the study's methods. The authors concluded that the use of multidisciplinary notes improved care.

The views expressed by respondents are in line with our recent study which showed that preprinted prompts led to admission notes that are both faster to write and more complete. ${ }^{1}$ Critics of preprinted prompts sometimes express concerns about clerking becoming mechanical and doctors not using their brains. We stress that preprinted prompts are merely the starting point and that appropriate extra details should be added in the space provided.

The views of our colleagues mean that we shall not be returning to traditional notes. The next version of our joint notes will feature fewer prompts and leave more space for freehand text. We recommend joint notes with preprinted prompts to others.

1 Goodyear HM, Lloyd BW. Can admission notes be improved by preprinted assessment sheets? Quality in Health Care 1995;4:190-3.

2 Williams JG, Roberts $R$, Rigby MJ. Integrated patient records: another move towards quality for patients. Quality in Health Care 1993;2:73-4.

3 D'Sa S. Multidisciplinary bedside notes: an experiment in care. Nursing Times 1995;91:46-7.

Appendix: Children's Department Joint* Medical and Nursing Notes Questionnaire for staff 5.5 .95

1 About you: Nurse Sister $\square$ Staff $\square$

Post Reg Student $\square$

RSCN $\square$ RSCN learner $\square$

Pre Reg Student Nurse $\square$ NNEB $\square$

Doctor Cons $\square \quad$ Reg $\square \quad$ SHO $\square$ Medical Student $\square$

2 About the notes:

Do you like the preprinted aspect of the notes? Yes $\square$ No $\square$ Not sure $\square$

Do you like the joint ${ }^{*}$ aspect of these notes? Yes $\square$ No $\square$ Not sure $\square$

Please list 3 features of the notes that are good, regarding preprinted aspects:

Please list 3 features of the notes that are bad, regarding preprinted aspects:

Please list 3 features of the notes that are good, regarding the joint ${ }^{\star}$ aspects:

Please list 3 features of the notes that are bad, regarding the joint ${ }^{*}$ aspects:

IF YOU were in charge (eg Senior Nurse or Clinical Director) what would your opinion be about how nurses and doctors record information about children on the wards.

* Would you keep the joint ${ }^{\star}$ notes as a good innovation?

Yes $\square$ No $\square$ Not sure $\square$

* Return to the previous system of separate notes? Yes $\square$ No $\square$ Not sure $\square$

Finally we would welcome any of your comments.

Thank you for your time. Please return them to me (.............) or put them in the envelopes attached to the ward notes trolleys for me to collect. 